\title{
Energy Efficient Clustering for Heterogeneous Wireless Sensor Networks - A Survey
}

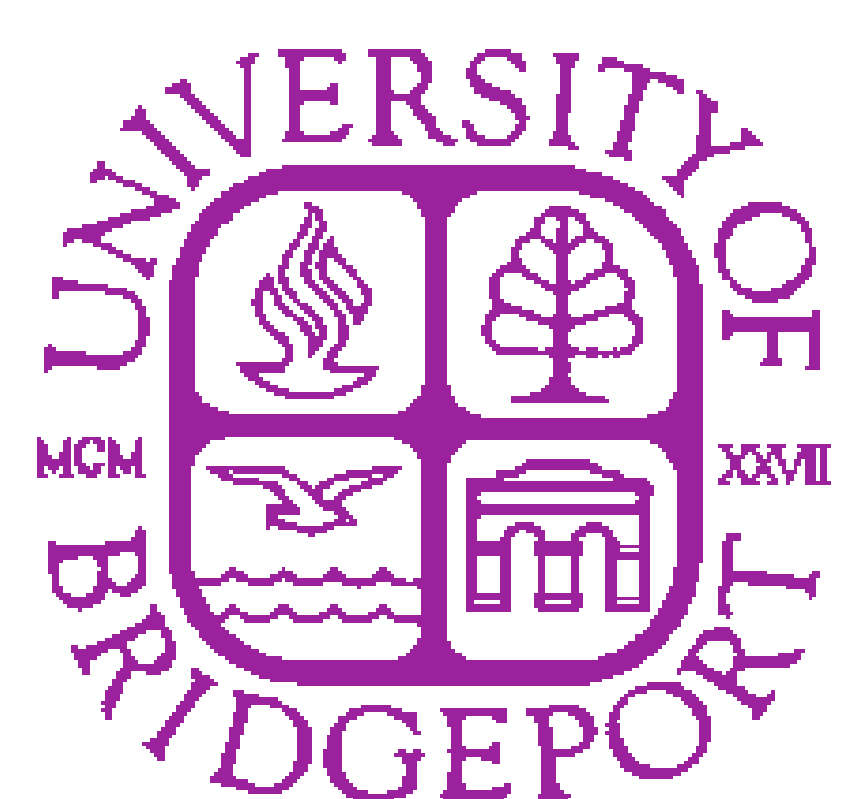

Baidar Ahmed Khan, Mohamed Ben Haj Frej, Linfeng Zhang

Department of Electrical Engineering

University of Bridgeport, Bridgeport, CT

\section{Abstract}

Ongoing advancements in electro-mechanical, power electronics and wireless communications have made possible the use of wireless sensor networks in remote applications such as environmental conditions, forest fire protection and target monitoring in military applications. These unattended applications require a huge number of sensor nodes working efficiently together for a common goal. Since sensor nodes have limited energy resources, it is of vital importance to use them in an efficient manner to improve a network's lifetime. One of the most important methods to achieve this is by making clusters. Nodes may not have the same energy, which makes it even more important for these clusters to work efficiently together in order to save power. Wireless sensor networks are of homogeneous and heterogeneous type. This paper presents a survey on energy efficient clustering mechanisms in heterogeneous wireless sensor networks. Nodes in homogeneous wireless sensor networks have similar resources, however heterogeneous networks have nodes with different energy levels that can be used effectively to enhance a network's lifetime.

\section{Introduction}

The popularity of wireless sensor networks is increasing because they can now be used in harsh and remote applications, such as disaster management, forest fire protection and habitat monitoring. These applications require a selfsustained energy efficient power system. Nodes are equipped with limited battery resources and it is not feasible to replace them or their batteries. A wireless sensor network contains multifunctional nodes deployed in an area of interest working towards a common goal. These low-cost sensor nodes have very limited computational, power and sensing resources. Due to the nature of their constrained resources, these networks should have a wise resource usage plan in order to enhance a network's lifetime, stability and reliability. Considerable research has been conducted and many techniques have been devised to elevate a network's lifetime.

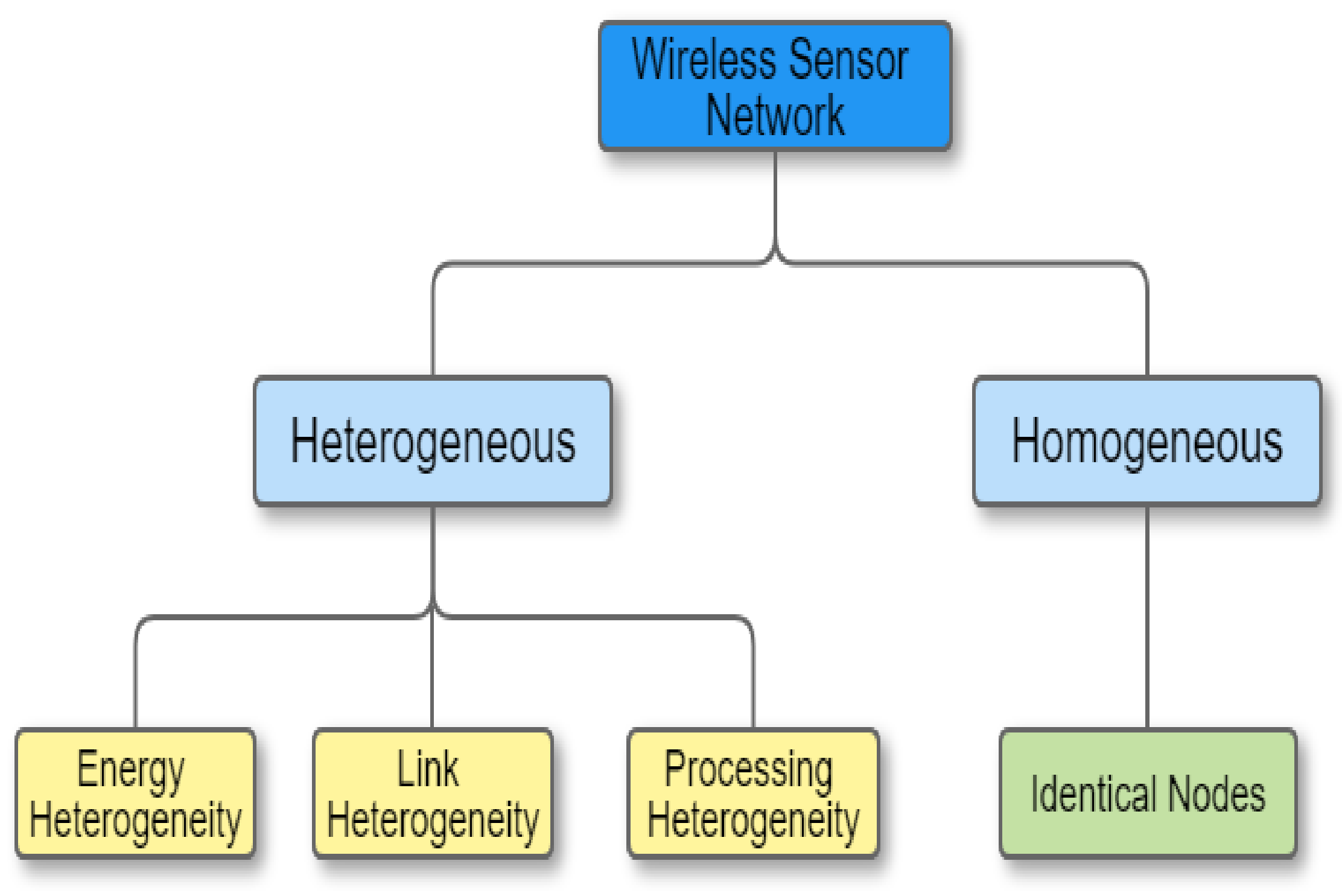

Figure 1: Wireless Sensor Network

Clustering is one of the best mechanisms to save energy and enhance a network's lifetime. Nodes are grouped together to form a cluster and a leader (cluster head) is elected. Cluster heads deliver useful information to the sink, so all nodes do not have to, giving immense energy savings. Wireless sensor networks are more heterogeneous in nature than homogeneous. Energy efficient methods for clustering need to be designed for heterogeneous sensor networks because clustering schemes designed for homogeneous networks do not work well with heterogeneous networks.

\section{Clustering and Cluster Head} Selection Criteria

Since nodes must communicate

to each other in order

to complete a task, they could end up utilizing most of their energy by continuously sending duplicate data. Many techniques and methods have been devised to address this problem One such method is known as clustering. A few to many nodes are grouped together to form a cluster. In every cluster, a suitable cluster

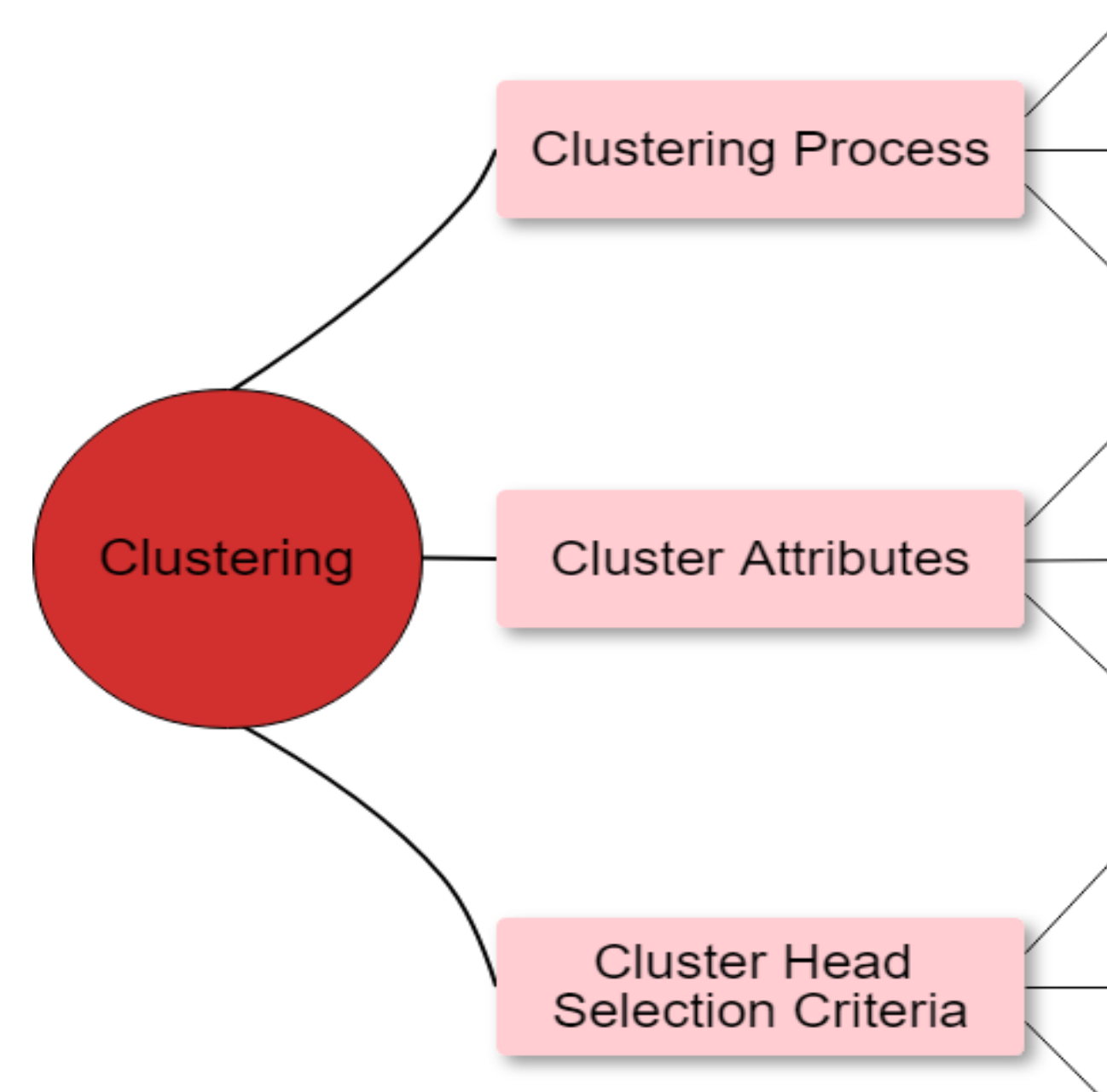

\section{Recapitulation of Studied Methods}

A variety of energy efficient clustering algorithms have been devised for heterogeneous wireless sensor networks. Saini and Sharma presented Threshold Distributed Energy Efficient Clustering Protocol (TDEEC) in which threshold is adjusted of the cluster head depending on the ratio of residual and average energy of a round. Elbhiri, Saadane and Aboutajdine presented Developed Distributed Energy-Efficient Clustering (DDEEC) in which probability of selection of cluster head is achieved with better efficiency. It is a distributed clustering algorithm in which cluster head selection is based on a node's initial and residual energy. Enhanced and Stochastic DEEC protocols work in the similar manner where initial and residual energy is used for the selection of cluster head.

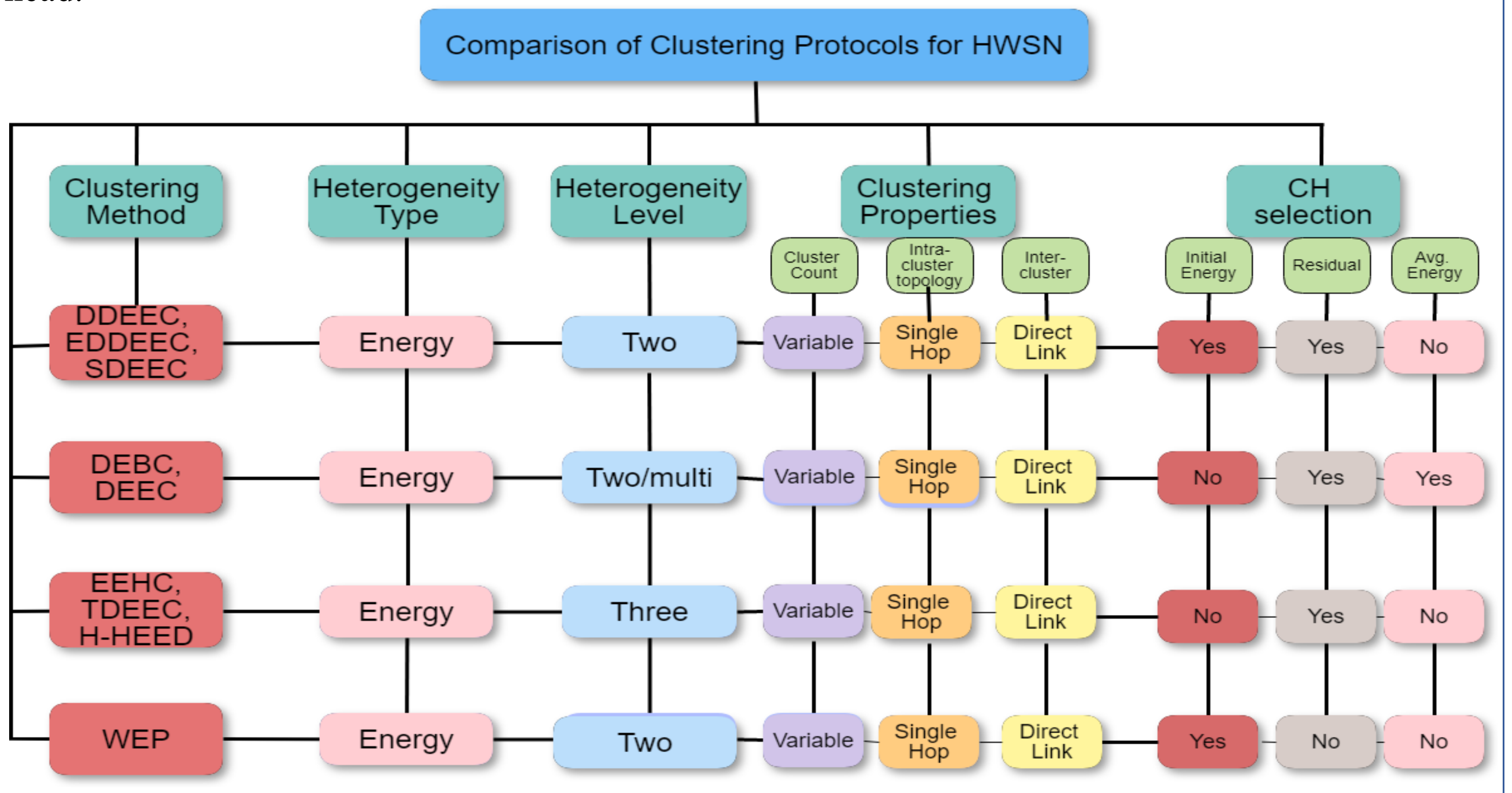

Figure 3: Comparison table of clustering protocols for heterogeneous wireless sensor networks

Duan and Fan presented Distributed Energy Balance Clustering Protocol (DEBC) in which cluster heads are selected based on the ratio of node's remaining energy and the average energy of the network. Nodes that have high residual and initial energy are more likely to become cluster heads. Kumar and Patel presented Energy Efficient Heterogeneous Clustered Scheme (EEHC) that elects a cluster head in a hierarchical based wireless sensor network. The probability of election is based on the residual energy of a node compared to other nodes in the network.

\section{Performance Measurement}

Performance measurement for heterogeneity can be divided into four main categories:

Network lifetime: It is the time when an operation starts until the time when the first node expires in the network.

No. of cluster heads in one round: All the nodes that will send data directly to BS.

No. of alive nodes in one round: All the nodes that still have some energy remaining and are not dead.

Throughput: This is the rate of data transmitted over the network.
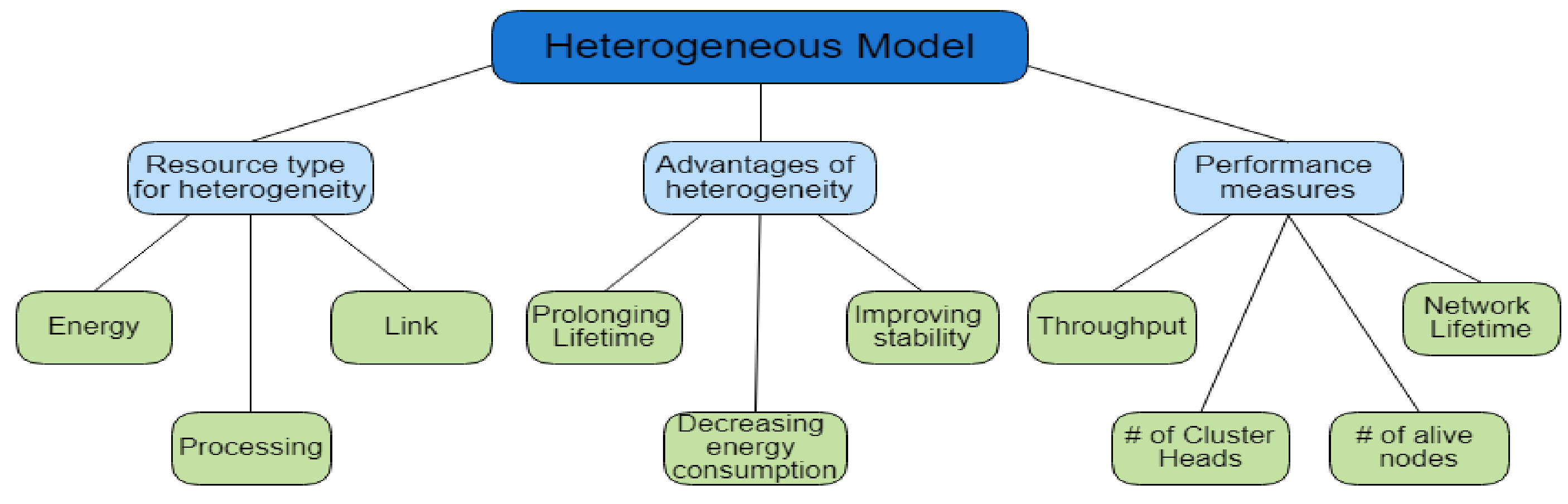

Figure 4: Heterogeneous model for WSN

\section{Conclusion}

Wireless sensor networks are used in harsh and remote applications such as fire prevention in forests, environmental monitoring and military surveillance of a target. These applications require efficient protocols in order to enhance a network's lifetime and stability because it is mostly not feasible to change nodes' batteries. Wireless sensor networks are realistically more heterogeneous in nature than homogeneous. Heterogeneity in the network can improve its lifetime and reliability Clustering is considered a useful method to enhance a network's efficiency in terms of stability and lifetime. This paper presents a survey on energy efficient clustering in heterogeneous wireless sensor networks and made a tabulated comparison between them

\section{References}

1. Abbasi, A.A. and M. Younis, A survey on clustering algorithms for wireless sensor networks Computer communications, 2007. 30(14-15): p. 2826-2841.

2. Akyildiz, I.F., et al., Wireless sensor networks: a survey. Computer networks, 2002. 38(4): p. 393-422

. Yick, J., B. Mukherjee, and D. Ghosal, Wireless sensor network survey. Computer networks, 2008. 52(12): p. 2292-2330.

4. Kumar, D., T.C. Aseri, and R. Patel, EEHC: Energy efficient heterogeneous clustered scheme for wireless sensor networks. computer communications, 2009. 32(4): p. 662-667. 